\title{
LOG-SINE EVALUATIONS OF MAHLER MEASURES
}

\author{
JONATHAN M. BORWEIN and ARMIN STRAUB ${ }^{\bowtie}$
}

\author{
(Received 20 December 2010; accepted 16 January 2012)
}

Communicated by I. E. Shparlinski

This paper is dedicated to the memory of Alf van der Poorten

\begin{abstract}
We provide evaluations of several recently studied higher and multiple Mahler measures using logsine integrals. This is complemented with an analysis of generating functions and identities for logsine integrals which allows the evaluations to be expressed in terms of zeta values or more general polylogarithmic terms. The machinery developed is then applied to evaluation of further families of multiple Mahler measures.
\end{abstract}

2010 Mathematics subject classification: primary 33E20; secondary 33F10, 11R06.

Keywords and phrases: log-sine integrals, Mahler measure, multiple polylogarithms, multiple zeta values, Clausen functions.

\section{Preliminaries}

For $k$ functions $P_{1}, P_{2}, \ldots, P_{k}$ (typically Laurent polynomials) in $n$ variables the multiple Mahler measure, introduced in [16], is defined by

$$
\mu\left(P_{1}, P_{2}, \ldots, P_{k}\right):=\int_{0}^{1} \cdots \int_{0}^{1} \prod_{j=1}^{k} \log \left|P_{j}\left(e^{2 \pi i t_{1}}, \ldots, e^{2 \pi i t_{n}}\right)\right| d t_{1} d t_{2} \ldots d t_{n} .
$$

When $P=P_{1}=P_{2}=\cdots=P_{k}$ this devolves to the higher Mahler measure, $\mu_{k}(P)$, as introduced and examined in [16]. When $k=1$ both reduce to the standard (logarithmic) Mahler measure [11].

For a positive integer $n$, we consider the log-sine integrals defined by

$$
\operatorname{Ls}_{n}(\sigma):=-\int_{0}^{\sigma} \log ^{n-1}\left|2 \sin \frac{\theta}{2}\right| d \theta
$$

Borwein was supported in part by the Australian Research Council and the University of Newcastle.

(C) 2012 Australian Mathematical Publishing Association Inc. 1446-7887/2012 \$16.00 
and their moments for $k \geq 0$ given by

$$
\operatorname{Ls}_{n}^{(k)}(\sigma):=-\int_{0}^{\sigma} \theta^{k} \log ^{n-1-k}\left|2 \sin \frac{\theta}{2}\right| d \theta
$$

This is the notation used by Lewin [18, 19], and the integrals in (1.2) are usually referred to as generalized log-sine integrals. Note that in each case the modulus is not needed if $0 \leq \sigma \leq 2 \pi$. Various log-sine integral evaluations may be found in Lewin's book [18, Sections 7.6 and 7.9].

We observe that $\operatorname{Ls}_{1}(\sigma)=-\sigma$ and that $\operatorname{Ls}_{n}^{(0)}(\sigma)=\operatorname{Ls}_{n}(\sigma)$. In particular,

$$
\operatorname{Ls}_{2}(\sigma)=\mathrm{Cl}_{2}(\sigma):=\sum_{n=1}^{\infty} \frac{\sin (n \sigma)}{n^{2}}
$$

$\mathrm{Cl}_{2}$ is the Clausen function, which plays a prominent role below. Generalized Clausen functions will be introduced in (1.7).

REMARK 1.1. It is fitting, given the dedication of this article and volume, that Alf van der Poorten wrote the foreword to Lewin's 'bible' [18]. In fact, he enthusiastically mentions the evaluation

$$
-\mathrm{Ls}_{4}^{(1)}\left(\frac{\pi}{3}\right)=\frac{17}{6480} \pi^{4}
$$

and its relation with inverse central binomial sums. This will be explained in Example 2.5. Evaluations of log-sine integrals at $\pi / 3$ are discussed in Section 2.2.

ExAmple 1.2 (Two classical Mahler measures revisited). As we will have recourse to the methods used in this example, we reevaluate $\mu(1+x+y)$ and $\mu(1+x+y+z)$. The starting point is Jensen's formula:

$$
\int_{0}^{1} \log \left|\alpha+e^{2 \pi i t}\right| d t=\log (\max \{|\alpha|, 1\})
$$

To evaluate $\mu(1+x+y)$, we use (1.4) to obtain

$$
\mu(1+x+y)=\int_{1 / 6}^{5 / 6} \log (2 \sin (\pi y)) d y=\frac{1}{\pi} \operatorname{Ls}_{2}\left(\frac{\pi}{3}\right)=\frac{1}{\pi} \mathrm{Cl}_{2}\left(\frac{\pi}{3}\right)
$$

which is a form of Smyth's seminal 1981 result (see [11, Appendix 1]).

To evaluate $\mu(1+x+y+z)$, we follow Boyd [11, Appendix 1] and observe, on applying Jensen's formula, that for complex constants $a$ and $b$,

$$
\mu(a x+b)=\log |a| \vee \log |b| .
$$


Writing $w=y / z$, we see that

$$
\begin{aligned}
\mu(1+x+y+z) & =\mu(1+x+z(1+w))=\mu(\log |1+w| \vee \log |1+x|) \\
& =\frac{1}{\pi^{2}} \int_{0}^{\pi} d \theta \int_{0}^{\pi} \max \left\{\log \left(2 \sin \frac{\theta}{2}\right), \log \left(2 \sin \frac{t}{2}\right)\right\} d t \\
& =\frac{2}{\pi^{2}} \int_{0}^{\pi} d \theta \int_{0}^{\theta} \log \left(2 \sin \frac{\theta}{2}\right) d t \\
& =\frac{2}{\pi^{2}} \int_{0}^{\pi} \theta \log \left(2 \sin \frac{\theta}{2}\right) d \theta \\
& =-\frac{2}{\pi^{2}} \operatorname{Ls}_{3}^{(1)}(\pi)=\frac{7}{2} \frac{\zeta(3)}{\pi^{2}}
\end{aligned}
$$

The final result is again due originally to Smyth.

In the following developments,

$$
\operatorname{Li}_{a_{1}, \ldots, a_{k}}(z):=\sum_{n_{1}>\cdots>n_{k}>0} \frac{z^{n_{1}}}{n_{1}^{a_{1}} \cdots n_{k}^{a_{k}}}
$$

denotes the generalized polylogarithm as studied in [5] and in [2, Ch. 3]. For our purposes, $a_{1}, \ldots, a_{k}$ will be positive integers. For example,

$$
\operatorname{Li}_{2,1}(z)=\sum_{k=1}^{\infty} \frac{z^{k}}{k^{2}} \sum_{j=1}^{k-1} \frac{1}{j} .
$$

In particular, $\operatorname{Li}_{k}(z)=\sum_{n=1}^{\infty} z^{n} / n^{k}$ is the polylogarithm of order $k$ and

$$
\operatorname{Ti}_{k}(x):=\sum_{n=0}^{\infty}(-1)^{n} \frac{x^{2 n+1}}{(2 n+1)^{k}}
$$

the related inverse tangent of order $k$. We use the same notation for the analytic continuations of these functions.

Moreover, multiple zeta values (MZVs) are denoted by

$$
\zeta\left(a_{1}, \ldots, a_{k}\right):=\operatorname{Li}_{a_{1}, \ldots, a_{k}}(1) .
$$

Similarly, we consider the multiple Clausen functions $(\mathrm{Cl})$ and multiple Glaisher functions (Gl) of depth $k$ and weight $w=a_{1}+\cdots+a_{k}$ defined by

$$
\begin{aligned}
& \mathrm{Cl}_{a_{1}, \ldots, a_{k}}(\theta)= \begin{cases}\operatorname{Im~Li}_{a_{1}, \ldots, a_{k}}\left(e^{i \theta}\right) & \text { if } w \text { is even, } \\
\operatorname{Re}_{\operatorname{Li}_{a_{1}, \ldots, a_{k}}\left(e^{i \theta}\right)} \text { if } w \text { is odd, }\end{cases} \\
& \mathrm{Gl}_{a_{1}, \ldots, a_{k}}(\theta)= \begin{cases}\operatorname{Re}_{\mathrm{Li}_{a_{1}, \ldots, a_{k}}\left(e^{i \theta}\right)} \text { if } w \text { is even, } \\
\operatorname{Im~Li}_{a_{1}, \ldots, a_{k}}\left(e^{i \theta}\right) & \text { if } w \text { is odd. }\end{cases}
\end{aligned}
$$


As illustrated in (1.3) and later in (2.6), the Clausen and Glaisher functions alternate between being cosine and sine series with the parity of the dimension. Of particular importance will be the case where $\theta=\pi / 3$, which has also been considered in [5]. Note that (1.7) agrees with the definition of $\mathrm{Cl}_{2}$ given in (1.3).

To conclude this section we recall the following Kummer-type polylogarithm [5, 18], which has been exploited in [10] among other places:

$$
\lambda_{n}(x):=(n-2) ! \sum_{k=0}^{n-2} \frac{(-1)^{k}}{k !} \operatorname{Li}_{n-k}(x) \log ^{k}|x|+\frac{(-1)^{n}}{n} \log ^{n}|x|,
$$

so that

$$
\lambda_{1}\left(\frac{1}{2}\right)=\log 2, \quad \lambda_{2}\left(\frac{1}{2}\right)=\frac{1}{2} \zeta(2), \quad \lambda_{3}\left(\frac{1}{2}\right)=\frac{7}{8} \zeta(3),
$$

and $\lambda_{4}\left(\frac{1}{2}\right)$ is the first to reveal the presence of $\operatorname{Li}_{n}\left(\frac{1}{2}\right)$.

Our other notation and usage is largely consistent with that in [18] and that in the newly published [20] in which most of the requisite material is described. Finally, a recent elaboration of what is meant when we speak about evaluations and 'closed forms' is to be found in [7].

\section{Log-sine integrals at $\pi$ and $\pi / 3$}

The multiple Mahler measure

$$
\mu_{k}\left(1+x+y_{*}\right):=\mu\left(1+x+y_{1}, 1+x+y_{2}, \ldots, 1+x+y_{k}\right)
$$

was studied by Sasaki [22, (4.1)]. He uses Jensen's formula (1.4) to observe that

$$
\mu_{k}\left(1+x+y_{*}\right)=\int_{1 / 6}^{5 / 6} \log ^{k}\left|1-e^{2 \pi i t}\right| d t
$$

and so provides an evaluation of $\mu_{2}\left(1+x+y_{*}\right)$. Further, we obtain the following theorem immediately from (2.2) and the definition (1.1) of the log-sine integrals.

THeORem 2.1. For positive integers $k$,

$$
\mu_{k}\left(1+x+y_{*}\right)=\frac{1}{\pi} \operatorname{Ls}_{k+1}\left(\frac{\pi}{3}\right)-\frac{1}{\pi} \operatorname{Ls}_{k+1}(\pi) .
$$

In Sections 2.1 and 2.2 we will cultivate Theorem 2.1 by showing how to recursively evaluate the log-sine integrals at $\pi$ and $\pi / 3$ respectively. In view of Theorem 2.1 , this provides evaluations of all multiple Mahler measures $\mu_{k}\left(1+x+y_{*}\right)$ as is made explicit in Section 3.1.

Other Mahler measure evaluations given later in this paper will further involve the generalized log-sine integrals, defined in (1.2), at $\pi$. These are studied in Section 2.3. 
2.1. Log-sine integrals at $\pi$. First, [19, Equation (8)] provides

$$
\operatorname{Ls}_{n+2}(\pi)=(-1)^{n} n !\left(\pi \alpha(n+1)+\sum_{k=1}^{n-2} \frac{(-1)^{k}}{(k+1) !} \alpha(n-k) \operatorname{Ls}_{k+2}(\pi)\right)
$$

where $\alpha(m)=\left(1-2^{1-m}\right) \zeta(m)$. Note that $\alpha(1)=0$, while for $m \geq 2$,

$$
\alpha(m)=-\mathrm{Li}_{m}(-1)=\sum_{k=1}^{\infty} \frac{(-1)^{k+1}}{k^{m}} .
$$

This is a consequence of the exponential generating function [18, Equation (7.109)] for the requisite log-sine integrals:

$$
-\sum_{m=0}^{\infty} \operatorname{Ls}_{m+1}(\pi) \frac{x^{m}}{m !}=\pi \frac{\Gamma(1+x)}{\Gamma^{2}(1+x / 2)}=\pi\left(\begin{array}{c}
x \\
x / 2
\end{array}\right) .
$$

This will be revisited and explained in Section 4.1.

ExAmple 2.2 (Values of $\operatorname{Ls}_{n}(\pi)$ ). We have $\operatorname{Ls}_{2}(\pi)=0$ and

$$
\begin{aligned}
-\operatorname{Ls}_{3}(\pi) & =\frac{1}{12} \pi^{3}, \\
\operatorname{Ls}_{4}(\pi) & =\frac{3}{2} \pi \zeta(3), \\
-\operatorname{Ls}_{5}(\pi) & =\frac{19}{240} \pi^{5}, \\
\operatorname{Ls}_{6}(\pi) & =\frac{45}{2} \pi \zeta(5)+\frac{5}{4} \pi^{3} \zeta(3), \\
-\operatorname{Ls}_{7}(\pi) & =\frac{275}{1344} \pi^{7}+\frac{45}{2} \pi \zeta^{2}(3), \\
\operatorname{Ls}_{8}(\pi) & =\frac{2835}{4} \pi \zeta(7)+\frac{315}{8} \pi^{3} \zeta(5)+\frac{133}{32} \pi^{5} \zeta(3),
\end{aligned}
$$

and so forth. The fact that each integral is a multi-variable rational polynomial in $\pi$ and zeta values follows directly from the recursion (2.4). Alternatively, these values may be conveniently obtained from (2.5) by a computer algebra system, as the following snippet of MAPLE code demonstrates:

for $\mathrm{k}$ to 7 do

$$
\operatorname{simplify}(\operatorname{subs}(x=\mathbb{0}, \operatorname{diff}(P i * \operatorname{binomial}(x, x / 2), x \$ k))) \text { od }
$$

2.2. Log-sine integrals at $\pi / 3$. In this section, we turn to the log-sine integrals at $\pi / 3$. It is shown in [8] that the log-sine integrals $\operatorname{Ls}_{n}^{(k)}(\tau)$ can be evaluated in terms of zeta values with the addition of multiple Clausen and Glaisher functions at $\tau$. The 
gist of the technique originates with Fuchs ([14], [18, Section 7.10]). In the case where $\tau=\pi / 3$ the resulting evaluations usually allow considerable reductions. This is because the basic sixth root of unity $\omega=e^{i \pi / 3}$ satisfies $\bar{\omega}=\omega^{2}$. As a consequence, the $\log$-sine integrals $\operatorname{Ls}_{n}^{(k)}(\pi / 3)$ are more tractable than those at other values, which fact we illustrate next.

Example 2.3 (Reducibility). Proceeding as in [8], in addition to $\operatorname{Ls}_{n}^{(n-1)}(\tau)=-\tau^{n} / n$ and $\mathrm{Ls}_{2}(\tau)=\mathrm{Cl}_{2}(\tau)$,

$$
\begin{aligned}
-\mathrm{Ls}_{3}(\tau) & =2 \mathrm{Gl}_{2,1}(\tau)+\frac{1}{12} \tau\left(3 \pi^{2}-3 \pi \tau+\tau^{2}\right) \\
\mathrm{Ls}_{3}^{(1)}(\tau) & =\mathrm{Cl}_{3}(\tau)+\tau \mathrm{Cl}_{2}(\tau)-\zeta(3)
\end{aligned}
$$

as well as

$$
\begin{aligned}
& -\mathrm{Ls}_{4}(\tau)=-6 \mathrm{Cl}_{2,1,1}(\tau)+\frac{3}{2} \mathrm{Cl}_{4}(\tau)+\frac{3}{2}(\pi-\tau) \mathrm{Cl}_{3}(\tau)-\frac{3}{4}(\pi-\tau)^{2} \mathrm{Cl}_{2}(\tau)-\frac{3}{2} \pi \zeta(3), \\
& \operatorname{Ls}_{4}^{(1)}(\tau)=\frac{1}{180} \pi^{4}-\frac{1}{16} \tau^{4}+\frac{1}{6} \pi \tau^{3}-\frac{1}{8} \pi^{2} \tau^{2}-2 \mathrm{Gl}_{3,1}(\tau)-2 \tau \mathrm{Gl}_{2,1}(\tau), \\
& \operatorname{Ls}_{4}^{(2)}(\tau)=-2 \mathrm{Cl}_{4}(\tau)+2 \tau \mathrm{Cl}_{3}(\tau)+\tau^{2} \mathrm{Cl}_{2}(\tau) .
\end{aligned}
$$

In the case $\tau=\pi / 3$ these evaluations can be further reduced, as will be shown in Example 2.4. On the other hand, it appears that, for instance, $\mathrm{Gl}_{2,1}(\tau)$ is not reducible even for the special values $\tau=\pi / 2$ or $\tau=2 \pi / 3$. Here, reducible means expressible in terms of multi-zeta values and Glaisher (or Clausen) functions of the same argument and lower weight. Yet, $\mathrm{Gl}_{2,1}(2 \pi / 3)$ is reducible to one-dimensional polylogarithmic terms at different arguments, as will be shown in (4.7).

More generally, in [3] explicit reductions for all polylogarithms of weight four or less are given.

Example 2.4 (Values of $\mathrm{Ls}_{n}(\pi / 3)$ ). The following evaluations may be obtained with the help of the implementation ${ }^{1}$ accompanying [8]:

$$
\begin{aligned}
\operatorname{Ls}_{2}\left(\frac{\pi}{3}\right) & =\mathrm{Cl}_{2}\left(\frac{\pi}{3}\right), \\
-\operatorname{Ls}_{3}\left(\frac{\pi}{3}\right) & =\frac{7}{108} \pi^{3}, \\
\operatorname{Ls}_{4}\left(\frac{\pi}{3}\right) & =\frac{1}{2} \pi \zeta(3)+\frac{9}{2} \mathrm{Cl}_{4}\left(\frac{\pi}{3}\right), \\
-\operatorname{Ls}_{5}\left(\frac{\pi}{3}\right) & =\frac{1543}{19440} \pi^{5}-6 \mathrm{Gl}_{4,1}\left(\frac{\pi}{3}\right), \\
\operatorname{Ls}_{6}\left(\frac{\pi}{3}\right) & =\frac{15}{2} \pi \zeta(5)+\frac{35}{36} \pi^{3} \zeta(3)+\frac{135}{2} \mathrm{Cl}_{6}\left(\frac{\pi}{3}\right), \\
-\operatorname{Ls}_{7}\left(\frac{\pi}{3}\right) & =\frac{74369}{326592} \pi^{7}+\frac{15}{2} \pi \zeta(3)^{2}-135 \mathrm{Gl}_{6,1}\left(\frac{\pi}{3}\right),
\end{aligned}
$$

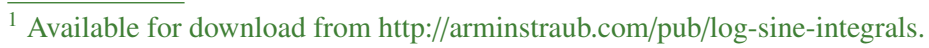




$$
\begin{aligned}
\operatorname{Ls}_{8}\left(\frac{\pi}{3}\right)=\frac{13181}{2592} \pi^{5} \zeta(3)+\frac{1225}{24} \pi^{3} \zeta(5)+\frac{319445}{864} \pi \zeta(7) \\
+\frac{35}{2} \pi^{2} \mathrm{Cl}_{6}\left(\frac{\pi}{3}\right)+\frac{945}{4} \mathrm{Cl}_{8}\left(\frac{\pi}{3}\right)+315 \mathrm{Cl}_{6,1,1}\left(\frac{\pi}{3}\right),
\end{aligned}
$$

and so forth, where we note that each integral is a multivariable rational polynomial in $\pi$ as well as $\mathrm{Cl}, \mathrm{Gl}$, and zeta values.

The first presumed-irreducible value that occurs is

$$
\begin{aligned}
\mathrm{Gl}_{4,1}\left(\frac{\pi}{3}\right) & =\sum_{n=1}^{\infty} \frac{\sum_{k=1}^{n-1} \frac{1}{k}}{n^{4}} \sin \left(\frac{n \pi}{3}\right) \\
& =\frac{3341}{1632960} \pi^{5}-\frac{1}{\pi} \zeta(3)^{2}-\frac{3}{4 \pi} \sum_{n=1}^{\infty} \frac{1}{\left(\begin{array}{c}
2 n \\
n
\end{array}\right) n^{6}} .
\end{aligned}
$$

The final evaluation is described in [5]. Extensive computation suggests it is not reducible in the sense of Example 2.3. Indeed, conjectures are made in [5, Section 5] for the number of irreducible Clausen and Glaisher values at each depth.

Example 2.5 (Central binomial sums). As suggested by (2.6), the log-sine integral $\operatorname{Ls}_{n}^{(1)}(\pi / 3)$ has an appealing evaluation in terms of the central binomial sum

$$
\mathcal{S}_{ \pm}(n):=\sum_{k=1}^{\infty} \frac{( \pm 1)^{k+1}}{\left(\begin{array}{c}
2 k \\
k
\end{array}\right) k^{n}}
$$

which is given by

$$
-\operatorname{Ls}_{n+2}^{(1)}\left(\frac{\pi}{3}\right)=n !\left(-\frac{1}{2}\right)^{n} \mathcal{S}_{+}(n+2) \text {. }
$$

This is proven in [5, Lemma 1], in connection with a study of Apéry-like sums, of which the value $\frac{5}{2} \mathcal{S}_{-}(3)=\zeta(3)$ plays a role in Apéry's proof of the latter's irrationality. The story of Apéry's proof is charmingly described in Alf van der Poorten's most cited paper [21].

Comtet's evaluation

$$
\mathcal{S}_{+}(4)=\frac{17}{36} \zeta(4)
$$

thus also evaluates

$$
\operatorname{Ls}_{4}^{(1)}\left(\frac{\pi}{3}\right)=-\frac{17 \pi^{4}}{6480}
$$

while the classical arcsin series gives $\operatorname{Ls}_{2}^{(1)}(\pi / 3)=-\pi^{2} / 18$. We recall from [5] that, for instance,

$$
\mathcal{S}_{+}(8)=\frac{3462601}{2204496000} \pi^{8}+\frac{1}{9} \pi^{2} \zeta(3)^{2}-\frac{38}{3} \zeta(3) \zeta(5)-\frac{14}{15} \zeta(5,3)-4 \pi \mathrm{Gl}_{6,1}\left(\frac{\pi}{3}\right) .
$$

Thus, apart from MZVs, $\mathcal{S}_{+}(8)$ involves the same Clausen value $\mathrm{Gl}_{6,1}(\pi / 3)$ as appears in $\operatorname{Ls}_{7}(\pi / 3)$ (and hence $\mu_{6}\left(1+x+y_{*}\right)$ ). In other words, $\mu_{6}\left(1+x+y_{*}\right)$ can be written entirely in terms of MZVs and $\mathcal{S}_{+}(8)$. This is true for the other cases in Example 3.1 as well: $\mu_{k}\left(1+x+y_{*}\right)$ can be written in terms of MZVs as well as $\mathcal{S}_{+}(k+2)$ for $k \leq 6$. 
2.3. Generalized log-sine integrals at $\boldsymbol{\pi}$. Following [8], we demonstrate how the generalized log-sine integrals $\operatorname{Ls}_{n}^{(k)}(\pi)$ may be extracted from a generating function given in Theorem 2.6. As Lewin [18, Section 7.9] sketches, at least for small values of $n$ and $k$, these log-sine integrals at $\pi$ have closed forms involving zeta values and Kummer-type constants such as $\mathrm{Li}_{4}(1 / 2)$. This will be made more precise in Remark 2.8. We start with the generating function identity:

$$
\begin{aligned}
-\sum_{n, k \geq 0} & \operatorname{Ls}_{n+k+1}^{(k)}(\pi) \frac{\lambda^{n}}{n !} \frac{(i \mu)^{k}}{k !} \\
= & \int_{0}^{\pi}\left(2 \sin \frac{\theta}{2}\right)^{\lambda} e^{i \mu \theta} d \theta \\
= & i e^{i \pi \lambda / 2} B(\mu-\lambda / 2,1+\lambda)-i e^{i \pi \mu} B_{1 / 2}(\mu-\lambda / 2,-\mu-\lambda / 2)
\end{aligned}
$$

given in [18]. Here $B_{x}$ is the incomplete beta function. With care (because of the singularities at zero), (2.8) can be differentiated as needed as suggested by Lewin.

Using the identities, valid for $a, b>0$ and $0<x<1$,

$$
B_{x}(a, b)=\frac{x^{a}(1-x)^{b-1}}{a}{ }_{2} F_{1}\left(\begin{array}{c}
1-b, 1 \\
a+1
\end{array} \mid \frac{x}{x-1}\right)=\frac{x^{a}(1-x)^{b}}{a}{ }_{2} F_{1}\left(\begin{array}{c}
a+b, 1 \\
a+1
\end{array} \mid x\right),
$$

found for instance in [20, Section 8.17(ii)], the generating function (2.8) may be rewritten as

$$
-\sum_{n, k \geq 0} \operatorname{Ls}_{n+k+1}^{(k)}(\pi) \frac{\lambda^{n}}{n !} \frac{(i \mu)^{k}}{k !}=i e^{i \pi \lambda / 2}\left(B_{1}(\mu-\lambda / 2,1+\lambda)-B_{-1}(\mu-\lambda / 2,1+\lambda)\right) .
$$

Upon expanding the right-hand side, this establishes the following computationally more accessible form given in [8].

Theorem 2.6 (Generating function for $\operatorname{Ls}_{n+k+1}^{(k)}(\pi)$ ). For $2|\mu|<\lambda<1$,

$$
-\sum_{n, k \geq 0} \operatorname{Ls}_{n+k+1}^{(k)}(\pi) \frac{\lambda^{n}}{n !} \frac{(i \mu)^{k}}{k !}=i \sum_{n \geq 0}(-1)^{n}\left(\begin{array}{l}
\lambda \\
n
\end{array}\right) \frac{e^{i \pi \lambda / 2}-(-1)^{n} e^{i \pi \mu}}{\mu-\lambda / 2+n} .
$$

The log-sine integrals $\operatorname{Ls}_{n}^{(k)}(\pi)$ can be quite comfortably extracted from (2.9) by appropriately differentiating its right-hand side. For that purpose it is very helpful to observe that

$$
\left.\frac{(-1)^{\alpha}}{\alpha !}\left(\frac{d}{d \lambda}\right)^{\alpha}\left(\begin{array}{l}
\lambda \\
n
\end{array}\right)\right|_{\lambda=0}=\frac{(-1)^{n}}{n} \sum_{n>i_{1}>i_{2}>\cdots>i_{\alpha-1}} \frac{1}{i_{1} i_{2} \cdots i_{\alpha-1}}
$$

Fuller theoretical and computational details are given in [8, pp. 43-50]. The general process is now exemplified for the cases $\operatorname{Ls}_{4}^{(2)}(\pi)$ and $\operatorname{Ls}_{5}^{(1)}(\pi)$. 
EXAMPLE $2.7\left(\operatorname{Ls}_{4}^{(k)}(\pi)\right.$ and $\left.\operatorname{Ls}_{5}^{(k)}(\pi)\right)$. In order to find $\operatorname{Ls}_{4}^{(2)}(\pi)$ we differentiate (2.9) once with respect to $\lambda$ and twice with respect to $\mu$. To further simplify computation, we take advantage of the fact that the result will be real, which allows us to neglect imaginary parts:

$$
\begin{aligned}
-\operatorname{Ls}_{4}^{(2)}(\pi) & =\left.\frac{d^{2}}{d \mu^{2}} \frac{d}{d \lambda} i \sum_{n \geq 0}\left(\begin{array}{l}
\lambda \\
n
\end{array}\right) \frac{(-1)^{n} e^{i \pi \lambda / 2}-e^{i \pi \mu}}{\mu-\lambda / 2+n}\right|_{\lambda=\mu=0} \\
& =2 \pi \sum_{n \geq 1} \frac{(-1)^{n+1}}{n^{3}}=\frac{3}{2} \pi \zeta(3) .
\end{aligned}
$$

In the second step we were able to drop the term corresponding to $n=0$ because its contribution $-i \pi^{4} / 24$ is purely imaginary.

Similarly, writing $H_{n-1}^{(1,1)}=\sum_{n>n_{1}>n_{2}} 1 /\left(n_{1} n_{2}\right)$, we obtain $\operatorname{Ls}_{5}^{(1)}(\pi)$ as

$$
\begin{aligned}
-\mathrm{Ls}_{5}^{(1)}(\pi)= & \frac{3}{4} \sum_{n \geq 1} \frac{6\left(1-(-1)^{n}\right)}{n^{5}}-\frac{\pi^{2}}{n^{3}}+\frac{8\left(1-(-1)^{n}\right)}{n^{4}}\left(n H_{n-1}^{(1,1)}-H_{n-1}\right) \\
= & \frac{9}{2}\left(\zeta(5)-\mathrm{Li}_{5}(-1)\right)-\frac{3}{4} \pi^{2} \zeta(3) \\
& +6\left(\mathrm{Li}_{3,1,1}(1)-\mathrm{Li}_{3,1,1}(-1)-\mathrm{Li}_{4,1}(1)+\mathrm{Li}_{4,1}(-1)\right) \\
= & 2 \lambda_{5}\left(\frac{1}{2}\right)-\frac{3}{4} \pi^{2} \zeta(3)-\frac{93}{32} \zeta(5) .
\end{aligned}
$$

Here $\lambda_{5}$ is as defined in (1.8). Further such evaluations include

$$
\begin{aligned}
& -\operatorname{Ls}_{4}^{(1)}(\pi)=2 \lambda_{4}\left(\frac{1}{2}\right)-\frac{19}{8} \zeta(4), \\
& -\operatorname{Ls}_{5}^{(2)}(\pi)=4 \pi \lambda_{4}\left(\frac{1}{2}\right)-\frac{3}{40} \pi^{5}, \\
& -\operatorname{Ls}_{5}^{(3)}(\pi)=\frac{9}{4} \pi^{2} \zeta(3)-\frac{93}{8} \zeta(5) .
\end{aligned}
$$

$\operatorname{Ls}_{5}^{(2)}(\pi)$ was also evaluated in [18, Equation (7.145)], but the exact formula was not given correctly.

REMARK 2.8. From the form of (2.9) and (2.10) we can see that the log-sine integrals $\operatorname{Ls}_{n}^{(k)}(\pi)$ can be expressed in terms of $\pi$ and the polylogarithms $\operatorname{Li}_{n,\{1\}_{m}}( \pm 1)$. Further, the duality results in [4, Section 6.3, Example 2.4] show that the terms $\operatorname{Li}_{n,\{1\}_{m}}(-1)$ will produce explicit multi-polylogarithmic values at $1 / 2$.

The next example illustrates the rapidly growing complexity of these integrals, especially when compared to the evaluations given in Example 2.7.

Example $2.9\left(\operatorname{Ls}_{6}^{(k)}(\pi)\right.$ and $\left.\operatorname{Ls}_{7}^{(3)}(\pi)\right)$. Proceeding as in Example 2.7 and writing

$$
\mathrm{Li}_{a_{1}, \ldots, a_{n}}^{ \pm}=\mathrm{Li}_{a_{1}, \ldots, a_{n}}(1)-\mathrm{Li}_{a_{1}, \ldots, a_{n}}(-1)
$$


we find that

$$
\begin{aligned}
-\mathrm{Ls}_{6}^{(1)}(\pi)=- & 24 \mathrm{Li}_{3,1,1,1}^{ \pm}+24 \mathrm{Li}_{4,1,1}^{ \pm}-18 \mathrm{Li}_{5,1}^{ \pm}+12 \mathrm{Li}_{6}^{ \pm} \\
& +3 \pi^{2} \zeta(3,1)-3 \pi^{2} \zeta(4)+\frac{\pi^{6}}{480} \\
=\frac{43}{60} & \log ^{6} 2-\frac{7}{12} \pi^{2} \log ^{4} 2+9 \zeta(3) \log ^{3} 2 \\
& +\left(24 \mathrm{Li}_{4}\left(\frac{1}{2}\right)-\frac{1}{120} \pi^{4}\right) \log ^{2} 2 \\
& +\left(36 \mathrm{Li}_{5}\left(\frac{1}{2}\right)-\pi^{2} \zeta(3)\right) \log 2 \\
& +12 \operatorname{Li}_{5,1}\left(\frac{1}{2}\right)+24 \mathrm{Li}_{6}\left(\frac{1}{2}\right)-\frac{247}{10080} \pi^{6} \\
=2 & \lambda_{6}\left(\frac{1}{2}\right)-6 \mathrm{Li}_{5,1}(-1)-3 \zeta(3)^{2}-\frac{451}{10080} \pi^{6}
\end{aligned}
$$

In the first equality, the term $\pi^{6} / 480$ is the one corresponding to $n=0$ in (2.9). Similarly,

$$
\begin{aligned}
& -\operatorname{Ls}_{6}^{(2)}(\pi)=4 \pi \lambda_{5}\left(\frac{1}{2}\right)-\pi^{3} \zeta(3)-\frac{189}{16} \pi \zeta(5), \\
& -\operatorname{Ls}_{6}^{(3)}(\pi)=6 \pi^{2} \lambda_{4}\left(\frac{1}{2}\right)-12 \operatorname{Li}_{5,1}(-1)-6 \zeta(3)^{2}-\frac{187}{1680} \pi^{6}, \\
& -\operatorname{Ls}_{6}^{(4)}(\pi)=-\frac{45}{2} \pi \zeta(5)+3 \pi^{3} \zeta(3),
\end{aligned}
$$

as well as

$$
\begin{aligned}
-\mathrm{Ls}_{7}^{(3)}(\pi)=\frac{9}{35} & \log ^{7} 2+\frac{4}{5} \pi^{2} \log ^{5} 2+9 \zeta(3) \log ^{4} 2-\frac{31}{30} \pi^{4} \log ^{3} 2 \\
& -\left(72 \operatorname{Li}_{5}\left(\frac{1}{2}\right)-\frac{9}{8} \zeta(5)-\frac{51}{4} \pi^{2} \zeta(3)\right) \log ^{2} 2 \\
& +\left(72 \operatorname{Li}_{5,1}\left(\frac{1}{2}\right)-216 \operatorname{Li}_{6}\left(\frac{1}{2}\right)+36 \pi^{2} \operatorname{Li}_{4}\left(\frac{1}{2}\right)\right) \log 2 \\
& +72 \operatorname{Li}_{6,1}\left(\frac{1}{2}\right)-216 \operatorname{Li}_{7}\left(\frac{1}{2}\right)+36 \pi^{2} \operatorname{Li}_{5}\left(\frac{1}{2}\right) \\
& -\frac{1161}{32} \zeta(7)-\frac{375}{32} \pi^{2} \zeta(5)+\frac{1}{10} \pi^{4} \zeta(3) \\
=6 & \pi^{2} \lambda_{5}\left(\frac{1}{2}\right)+36 \operatorname{Li}_{5,1,1}(-1)-\pi^{4} \zeta(3)-\frac{759}{32} \pi^{2} \zeta(5)-\frac{45}{32} \zeta(7) .
\end{aligned}
$$

Note that in each case the monomials in $\operatorname{Ls}_{n}^{(k)}(\pi)$ are of total order $n$, where $\pi$ is order one, $\zeta(3)$ is order three and so on.

REMARK 2.10. A purely real form of Theorem 2.6 is the following:

$$
\int_{0}^{\pi}\left(2 \sin \frac{\theta}{2}\right)^{x} e^{\theta y} d \theta=\sum_{n=0}^{\infty} \frac{(-1)^{n}\left(\begin{array}{l}
x \\
n
\end{array}\right)\left(y\left((-1)^{n} e^{\pi y}-\cos \frac{\pi x}{2}\right)-\left(n-\frac{x}{2}\right) \sin \frac{\pi x}{2}\right)}{\left(n-\frac{x}{2}\right)^{2}+y^{2}} .
$$

One may now also deduce one-variable generating functions from (2.16). For instance,

$$
\sum_{n=0}^{\infty} \operatorname{Ls}_{n+2}^{(1)}(\pi) \frac{\lambda^{n}}{n !}=\sum_{n=0}^{\infty}\left(\begin{array}{l}
\lambda \\
n
\end{array}\right) \frac{(-1)^{n} \cos (\pi \lambda / 2)-1}{(n-\lambda / 2)^{2}}
$$

and we may again now extract individual values. 
2.4. Hypergeometric evaluation of $\operatorname{Ls}_{\boldsymbol{n}}(\boldsymbol{\pi} / \mathbf{3})$. We close this section with an alternative approach to the evaluation of $\operatorname{Ls}_{n}(\pi / 3)$ complementing the one given in Section 2.2.

Theorem 2.11 (Hypergeometric form of $\operatorname{Ls}_{n}(\pi / 3)$ ). For nonnegative integers $n$,

$$
\frac{(-1)^{n+1}}{n !} \operatorname{Ls}_{n+1}\left(\frac{\pi}{3}\right)={ }_{n+2} F_{n+1}\left(\begin{array}{c|c}
\{1 / 2\}^{n+2} & 1 \\
\{3 / 2\}^{n+1} & \frac{1}{4}
\end{array}\right)=\sum_{k=0}^{\infty} \frac{2^{-4 k}}{(2 k+1)^{n+1}}\left(\begin{array}{c}
2 k \\
k
\end{array}\right) .
$$

Consequently,

$$
-\sum_{n=0}^{\infty} \operatorname{Ls}_{n+1}\left(\frac{\pi}{3}\right) \frac{s^{n}}{n !}=\frac{1}{s+1}{ }_{2} F_{1}\left(\begin{array}{c|c}
\frac{1}{2}, \frac{s}{2}+\frac{1}{2} & \frac{1}{4} \\
\frac{s}{2}+\frac{3}{2} & 4
\end{array}\right)=\sum_{k=0}^{\infty} \frac{2^{-4 k}}{2 k+1+s}\left(\begin{array}{c}
2 k \\
k
\end{array}\right) .
$$

Proof. We compute as follows:

$$
\begin{aligned}
-\operatorname{Ls}_{n+1}\left(\frac{\pi}{3}\right) & =\int_{0}^{\pi / 3} \log ^{n}\left(2 \sin \frac{\theta}{2}\right) d \theta \\
& =\int_{0}^{1} \frac{\log ^{n}(x)}{\sqrt{1-x^{2} / 4}} d x \\
& =\sum_{k=0}^{\infty} 2^{-4 k}\left(\begin{array}{c}
2 k \\
k
\end{array}\right) \int_{0}^{1} x^{2 k} \log ^{n}(x) d x .
\end{aligned}
$$

The claim thus follows from

$$
\int_{0}^{1} x^{s-1} \log ^{n}(x) d x=\int_{0}^{\infty}(-x)^{n} e^{-s x} d x=\frac{(-1)^{n} \Gamma(n+1)}{s^{n+1}},
$$

which is a consequence of the integral representation of the gamma function.

Observe that the sum in (2.17) converges very rapidly and so is very suitable for computation. Also, from Example 2.4 we have evaluations-some known-such as

$$
\sum_{k=0}^{\infty} \frac{2^{-4 k}}{(2 k+1)}\left(\begin{array}{c}
2 k \\
k
\end{array}\right)=\frac{\pi}{3}
$$

and

$$
\sum_{k=0}^{\infty} \frac{2^{-4 k}}{(2 k+1)^{2}}\left(\begin{array}{c}
2 k \\
k
\end{array}\right)=\mathrm{Cl}_{2}\left(\frac{\pi}{3}\right)
$$

ReMARK 2.12. As outlined in [12], the series (2.17) combined with (2.7) can also be used to produce rapidly-convergent series for certain multi-zeta values including $\zeta(5,3), \zeta(7,3)$ and $\zeta(3,5,3)$. 


\section{Log-sine evaluations of multiple Mahler measures}

We first substantiate our claim that we can recursively determine $\mu_{k}\left(1+x+y_{*}\right)$ from Equation (2.3).

3.1. Evaluation of $\boldsymbol{\mu}_{\boldsymbol{k}}\left(\mathbf{1}+\boldsymbol{x}+\boldsymbol{y}_{*}\right)$. Substituting the values given in Examples 2.4 and 2.2 into Equation (2.3) we obtain the following multiple Mahler evaluations.

Example 3.1 (Values of $\mu_{k}\left(1+x+y_{*}\right)$ ). We have

$$
\begin{aligned}
& \mu_{1}\left(1+x+y_{*}\right)=\frac{1}{\pi} \mathrm{Cl}_{2}\left(\frac{\pi}{3}\right), \\
& \mu_{2}\left(1+x+y_{*}\right)=\frac{\pi^{2}}{54}, \\
& \mu_{3}\left(1+x+y_{*}\right)=\frac{9}{2 \pi} \mathrm{Cl}_{4}\left(\frac{\pi}{3}\right)-\zeta(3), \\
& \mu_{4}\left(1+x+y_{*}\right)=\frac{6}{\pi} \mathrm{Gl}_{4,1}\left(\frac{\pi}{3}\right)-\frac{\pi^{4}}{4860}, \\
& \mu_{5}\left(1+x+y_{*}\right)=\frac{135}{2 \pi} \mathrm{Cl}_{6}\left(\frac{\pi}{3}\right)-15 \zeta(5)-\frac{5}{18} \pi^{2} \zeta(3), \\
& \mu_{6}\left(1+x+y_{*}\right)=\frac{135}{\pi} \operatorname{Gl}_{6,1}\left(\frac{\pi}{3}\right)+15 \zeta(3)^{2}-\frac{943}{40824} \pi^{6},
\end{aligned}
$$

and the like. The first is again a form of Smyth's result (1.5).

Remark 3.2. Note that we may rewrite the multiple Mahler measure $\mu_{k}\left(1+x+y_{*}\right)$ as follows:

$$
\mu_{k}\left(1+x+y_{*}\right)=\mu(\underbrace{1+x, \ldots, 1+x}_{k-1}, 1+x+y) .
$$

This is easily seen from Jensen's formula (1.4). Indeed, using (1.4), the left-hand side of (3.2) becomes

$$
\begin{aligned}
\mu_{k}\left(1+x+y_{*}\right) & =\int_{0}^{1} \ldots \int_{0}^{1} \prod_{j=1}^{k} \log \left|1+e^{2 \pi i s}+e^{2 \pi i t_{j}}\right| d s d t_{1} \ldots d t_{k} \\
& =\int_{0}^{1}\left[\int_{0}^{1} \log \left|1+e^{2 \pi i s}+e^{2 \pi i t}\right| d t\right]^{k} d s \\
& =\int \log ^{k}\left|1+e^{2 \pi i s}\right| d s
\end{aligned}
$$

where the last integral is over $s$ in $[0,1]$ such that $\left|1+e^{2 \pi i s}\right| \geq 1$. The same integral is obtained when applying (1.4) to the right-hand side of (3.2). 
3.2. Evaluation of $\boldsymbol{\mu}_{k}\left(\mathbf{1}+\boldsymbol{x}+\boldsymbol{y}_{*}+z_{*}\right)$. We next follow a similar course for multiple Mahler measures built from $1+x+y+z$ to that given for $\mu_{k}\left(1+x+y_{*}\right)$ in Section 3.1. Analogous to (2.1) we define:

$$
\mu_{k}\left(1+x+y_{*}+z_{*}\right):=\mu\left(1+x+y_{1}+z_{1}, \ldots, 1+x+y_{k}+z_{k}\right) .
$$

Working as in (1.6), we may write

$$
\mu_{k}\left(1+x+y_{*}+z_{*}\right)=\frac{1}{\pi} \int_{0}^{\pi}\left[\frac{1}{\pi} \int_{0}^{\pi} \max \left\{\log \left(2 \sin \frac{\theta}{2}\right), \log \left(2 \sin \frac{\sigma}{2}\right)\right\} d \sigma\right]^{k} d \theta .
$$

We observe that the inner integral with respect to $\sigma$ evaluates separately, and on recalling that $\mathrm{Ls}_{2}(\theta)=\mathrm{Cl}_{2}(\theta)$ and $\mathrm{Cl}_{2}(\pi)=0$, we arrive at our next theorem.

Theorem 3.3. For all positive integers $k$,

$$
\mu_{k}\left(1+x+y_{*}+z_{*}\right)=\frac{1}{\pi^{k+1}} \int_{0}^{\pi}\left(\theta \log \left(2 \sin \frac{\theta}{2}\right)+\mathrm{Cl}_{2}(\theta)\right)^{k} d \theta .
$$

Example 3.4 (Values of $\mu_{k}\left(1+x+y_{*}+z_{*}\right)$ ). Thus, for $\mu_{2}\left(1+x+y_{*}+z_{*}\right.$ ),

$$
\pi^{3} \mu_{2}\left(1+x+y_{*}+z_{*}\right)=-\mathrm{Ls}_{5}^{(2)}(\pi)+\int_{0}^{\pi} \mathrm{Cl}_{2}^{2}(\theta) d \theta+\int_{0}^{\pi} 2 \theta \log \left(2 \sin \frac{\theta}{2}\right) \mathrm{Cl}_{2}(\theta) d \theta .
$$

Applying Parseval's equation evaluates the first integral in this equation to $\pi^{5} / 180$. Integration by parts of the second integral shows that it equals minus the first one.

For $k=3$, one term is a log-sine integral and two of the terms are equal, but we could not completely evaluate the two remaining terms.

Hence, from (3.3),

$$
\begin{gathered}
\mu_{1}\left(1+x+y_{*}+z_{*}\right)=-\frac{2}{\pi^{2}} \operatorname{Ls}_{3}^{(1)}(\pi)=\frac{7}{2} \frac{\zeta(3)}{\pi^{2}}, \\
\mu_{2}\left(1+x+y_{*}+z_{*}\right)=-\frac{1}{\pi^{3}} \operatorname{Ls}_{5}^{(2)}(\pi)+\frac{\pi^{2}}{90}=\frac{4}{\pi^{2}} \operatorname{Li}_{3,1}(-1)+\frac{7}{360} \pi^{2},
\end{gathered}
$$

and

$$
\begin{aligned}
\mu_{3}(1+ & \left.x+y_{*}+z_{*}\right) \\
= & \frac{2}{\pi^{4}} \int_{0}^{\pi} \mathrm{Cl}_{2}^{3}(\theta) d \theta+\frac{3}{\pi^{4}} \int_{0}^{\pi} \theta^{2} \log ^{2}\left(2 \sin \frac{\theta}{2}\right) \mathrm{Cl}_{2}(\theta) d \theta \\
& -\frac{1}{\pi^{4}} \operatorname{Ls}_{7}^{(3)}(\pi) .
\end{aligned}
$$

The first of these is a form of (1.6) which originates with Smyth and Boyd [11]. The relevant $\log$-sine integrals have been discussed in Section 2.3. In particular, $\operatorname{Ls}_{5}^{(2)}(\pi)$ and $\operatorname{Ls}_{7}^{(3)}(\pi)$ have been evaluated in (2.13) and (2.15).

It is possible to further reexpress the integrals in (3.4), but we have not so far found an entirely satisfactory resolution. 
3.3. Evaluation of $\boldsymbol{\mu}(\mathbf{1}+\boldsymbol{x}, \ldots, \mathbf{1}+\boldsymbol{x}, \mathbf{1}+\boldsymbol{x}+\boldsymbol{y}+\boldsymbol{z})$. By Remark 3.2, the multiple Mahler measure $\mu_{k}\left(1+x+y_{*}\right)$ may be rewritten as $\mu(1+x, \ldots, 1+x, 1+$ $x+y)$, with the term $1+x$ repeated $k-1$ times. This is not possible for $\mu_{k}\left(1+x+y_{*}+\right.$ $\left.z_{*}\right)$, which is distinct from $\mu(1+x, \ldots, 1+x, 1+x+y+z)$, which we study next.

Applying Jensen's formula as in (1.6) for $k=0,1,2, \ldots$, we obtain (3.5) below. Then (3.6) follows on integrating by parts.

Theorem 3.5. For all nonnegative integers $k$,

$$
\begin{aligned}
\mu(1+ & x, \ldots, 1+x, 1+x+y+z) \\
& =-\frac{1}{\pi^{2}} \operatorname{Ls}_{k+3}^{(1)}(\pi)+\frac{1}{\pi^{2}} \int_{0}^{\pi} \operatorname{Ls}_{2}(\theta) \log ^{k}\left(2 \sin \frac{\theta}{2}\right) d \theta \\
& =-\frac{1}{\pi^{2}} \operatorname{Ls}_{k+3}^{(1)}(\pi)-\frac{1}{\pi^{2}} \int_{0}^{\pi} \operatorname{Ls}_{k+1}(\theta) \log \left(2 \sin \frac{\theta}{2}\right) d \theta,
\end{aligned}
$$

where $\mu$ has $k+2$ arguments, of which $k+1$ are exactly $1+x$.

Example 3.6. Equation (3.6) recovers (1.6) when $k=0$ since $\operatorname{Ls}_{1}(\theta)=-\theta$. Setting $k=1$ in (3.6), we obtain

$$
\begin{aligned}
\mu(1+x, 1+x+y+z) & =-\frac{1}{\pi^{2}} \operatorname{Ls}_{4}^{(1)}(\pi)+\frac{1}{\pi^{2}} \int_{0}^{\pi} \mathrm{Cl}_{2}(\theta) \log \left(2 \sin \frac{\theta}{2}\right) d \theta \\
& =-\frac{1}{\pi^{2}} \operatorname{Ls}_{4}^{(1)}(\pi)-\frac{1}{2 \pi^{2}} \mathrm{Cl}_{2}^{2}(\pi) \\
& =-\frac{1}{\pi^{2}} \operatorname{Ls}_{4}^{(1)}(\pi)=\frac{2}{\pi^{2}} \lambda_{4}\left(\frac{1}{2}\right)-\frac{19}{720} \pi^{2}
\end{aligned}
$$

on again using $\operatorname{Ls}_{2}(\theta)=\mathrm{Cl}_{2}(\theta)$ and $\mathrm{Cl}_{2}(\pi)=0$. The final evaluation was given in (2.12) of Example 2.7. For $k=2$,

$$
\begin{aligned}
\mu(1+x, 1+x, 1+x+y+z) & =-\frac{1}{\pi^{2}} \operatorname{Ls}_{5}^{(1)}(\pi)+\frac{1}{\pi^{2}} \int_{0}^{\pi} \operatorname{Ls}_{2}(\theta) \log ^{2}\left(2 \sin \frac{\theta}{2}\right) d \theta \\
& =-\frac{1}{\pi^{2}} \operatorname{Ls}_{5}^{(1)}(\pi)-\frac{2}{3 \pi^{2}} \lambda_{5}\left(\frac{1}{2}\right)+\frac{155}{32 \pi^{2}} \zeta(5),
\end{aligned}
$$

where the last integral was found via the integer relation algorithm PSLQ. This agrees with the more complicated version conjectured in [15]. We may use (2.11) of Example 2.7 to arrive at

$$
\mu(1+x, 1+x, 1+x+y+z)=\frac{4}{3 \pi^{2}} \lambda_{5}\left(\frac{1}{2}\right)-\frac{3}{4} \zeta(3)+\frac{31}{16 \pi^{2}} \zeta(5) .
$$

For $k=3$, things are more complicated, as is suggested by (2.14).

\section{Moments of random walks}

The $s$ th moments of an $n$-step uniform random walk are given by

$$
W_{n}(s)=\int_{0}^{1} \cdots \int_{0}^{1}\left|\sum_{k=1}^{n} e^{2 \pi i t_{k}}\right|^{s} d t_{1} \cdots d t_{n},
$$


and their relation with Mahler measure is observed in [9]. In particular,

$$
W_{n}^{\prime}(0)=\mu\left(1+x_{1}+\cdots+x_{n-1}\right)
$$

with the cases $n=3$ and $n=4$ given in (1.5) and (1.6) respectively. The cases $n=5$ and $n=6$ are discussed in (4.18) and (4.19) respectively. Higher derivatives of $W_{n}$ correspond to higher Mahler measures:

$$
W_{n}^{(k)}(0)=\mu_{k}\left(1+x_{1}+\cdots+x_{n-1}\right) .
$$

More general moments corresponding to other Mahler measures were introduced in [1] and studied in [16] as zeta Mahler measures.

4.1. Evaluation of $\mu_{k}(1+x)$. Equipped with the results of the first section, we may now fruitfully revisit another recent result which is concerned with the evaluation of $W_{2}^{(k)}(0)=\mu_{k}(1+x)$.

A central evaluation in [16, Theorem 3] is

$$
\mu_{k}(1+x)=(-1)^{k} k ! \sum_{n=1}^{\infty} \frac{1}{4^{n}} \sum_{b_{j} \geq 2, \sum b_{j}=k} \zeta\left(b_{1}, b_{2}, \ldots, b_{n}\right) .
$$

We note that, directly from the definition and an easy change of variables,

$$
\mu_{k}(1+x)=-\frac{1}{\pi} \operatorname{Ls}_{k+1}(\pi) .
$$

Hence, we have closed forms such as provided by Example 2.2.

EXAMPLE 4.1. For instance,

$$
\begin{aligned}
-\mu_{5}(1+x) & =\frac{45}{2} \zeta(5)+\frac{5}{4} \pi^{2} \zeta(3), \\
\mu_{6}(1+x) & =\frac{45}{2} \zeta^{2}(3)+\frac{275}{1344} \pi^{6} .
\end{aligned}
$$

These are derived more elaborately in [16, Example 5] from the right-hand side of Equation (4.2).

We have, inter alia, evaluated the multi-zeta value sum on the right of Equation (4.2) as a simple log-sine integral.

Also, note that the evaluation $W_{2}(s)=\left(\begin{array}{c}s \\ s / 2\end{array}\right)$, given in [9], in combination with (4.3) thus explains and proves the generating function (2.5).

4.2. A generating function for $\boldsymbol{\mu}_{\boldsymbol{k}}(\mathbf{1}+\boldsymbol{x}+\boldsymbol{y})$. The evaluation of the Mahler measures $W_{3}^{\prime}(0)=\mu(1+x+y)$ and $W_{4}^{\prime}(0)=\mu(1+x+y+z)$ is classical and was discussed in Example 1.2.

The derivatives $W_{3}^{\prime \prime}(0)=\mu_{2}(1+x+y)$ and $W_{4}^{\prime \prime}(0)=\mu_{2}(1+x+y+z)$ were evaluated using explicit forms for $W_{3}(s)$ and $W_{4}(s)$ in [9, Section 6]. For example,

$$
W_{3}^{\prime \prime}(0)=\frac{\pi^{2}}{12}-\frac{4 \log 2}{\pi} \mathrm{Cl}_{2}\left(\frac{\pi}{3}\right)-\frac{4}{\pi} \sum_{n=0}^{\infty} 4^{-2 n}\left(\begin{array}{c}
2 n \\
n
\end{array}\right) \frac{1}{(2 n+1)^{2}} \sum_{k=0}^{n} \frac{1}{2 k+1} .
$$


We shall revisit these two Mahler measures in (4.6) and (4.17) of Sections 4.3 and 4.4.

As a consequence of the study of random walks in [9] we record the following generating function for $\mu_{k}(1+x+y)$ which follows from (4.1) and the hypergeometric expression for $W_{3}$ in [9, Theorem 10]. There is a corresponding expression, using a single Meijer $G$-function, for $W_{4}$ (that is, $\mu_{m}(1+x+y+z)$ ) given in [9, Theorem 11].

Theorem 4.2. For complex $|s|<2$, we may write

$$
\sum_{m=0}^{\infty} \mu_{m}(1+x+y) \frac{s^{m}}{m !}=W_{3}(s)=\frac{\sqrt{3}}{2 \pi} 3^{s+1} \frac{\Gamma(1+s / 2)^{2}}{\Gamma(s+2)}{ }_{3} F_{2}\left(\begin{array}{c}
\frac{s+2}{2}, \frac{s+2}{2}, \frac{s+2}{2} \\
1, \frac{s+3}{2}
\end{array} \mid \frac{1}{4}\right) .
$$

The particular measure $\mu_{2}(1+x+y)$ will be investigated in Section 4.3. The general case $\mu_{m}(1+x+y)$ is studied in [3].

\subsection{Evaluation of $\mu_{2}(1+x+y)$.}

EXAmple 4.3. A purported evaluation given in [16] is:

$$
\mu_{2}(1+x+y)=\mu_{2}(1+x+y) \stackrel{?}{=} \frac{5}{54} \pi^{2}=5 \mu_{2}\left(1+x+y_{*}\right)
$$

where the last equality follows from (3.1). However, we are able to numerically disprove $(4.4)^{1}$. Indeed, we find $\mu_{2}(1+x+y) \approx 0.419299$ while $5 \pi^{2} / 54 \approx 0.913852$.

We note that for integer $k \geq 1$ we do have

$$
\mu_{k}(1+x+y)=\frac{1}{4 \pi^{2}} \int_{0}^{2 \pi} d \theta \int_{0}^{2 \pi}\left(\operatorname{Re} \log \left(1-2 \sin (\theta) e^{i \omega}\right)\right)^{k} d \omega
$$

directly from the definition and some simple trigonometry, since Re $\log =\log |\cdot|$. We revisit Example 4.3 in the next result, in which we evaluate $\mu_{2}(1+x+y)$ as a log-sine integral as well as in terms of polylogarithmic constants.

THEOREM 4.4. We have

$$
\begin{aligned}
\mu_{2}(1+x+y) & =\frac{24}{5 \pi} \operatorname{Ti}_{3}\left(\frac{1}{\sqrt{3}}\right)+\frac{2 \log 3}{\pi} \mathrm{Cl}_{2}\left(\frac{\pi}{3}\right)-\frac{\log ^{2} 3}{10}-\frac{19 \pi^{2}}{180} \\
& =\frac{\pi^{2}}{4}+\frac{3}{\pi} \operatorname{Ls}_{3}\left(\frac{2 \pi}{3}\right)
\end{aligned}
$$

REMARK 4.5. We note that

$$
\operatorname{Ls}_{3}\left(\frac{2 \pi}{3}\right)=-\int_{0}^{\pi / 3} \log ^{2}\left(2 \cos \frac{\theta}{2}\right) d \theta
$$

\footnotetext{
$\overline{1}$ There are two errors in the proof given in [16, Theorem 11]. A term is dropped between lines 8 and 9 of the proof and the limits of integration are wrong after changing $s(1-s)$ to $t$.
} 
and that these log-cosine integrals have fewer explicit closed forms. Using the results of [8] to evaluate log-sine integrals in polylogarithmic terms we find that

$$
\operatorname{Ls}_{3}\left(\frac{2 \pi}{3}\right)=-\frac{13}{162} \pi^{3}-2 \mathrm{Gl}_{2,1}\left(\frac{2 \pi}{3}\right) \text {. }
$$

In fact, this is automatic if we employ the implementation provided with [8]. Theorem 4.4 thus also gives a reduction of $\mathrm{Gl}_{2,1}(2 \pi / 3)$ to one-dimensional polylogarithmic constants.

A preparatory result is helpful before proceeding to the proof of Theorem 4.4.

Proposition 4.6 (A dilogarithmic representation). The following equalities hold.

$$
\begin{aligned}
& \frac{2}{\pi} \int_{0}^{\pi} \operatorname{Re}^{2 i} i_{2}\left(4 \sin ^{2} \theta\right) d \theta=2 \zeta(2), \\
& \mu_{2}(1+x+y)=\frac{1}{36} \pi^{2}+\frac{2}{\pi} \int_{0}^{\pi / 6} \operatorname{Li}_{2}\left(4 \sin ^{2} \theta\right) d \theta .
\end{aligned}
$$

Proof. For (4.8), we define

$$
\tau(z):=\frac{2}{\pi} \int_{0}^{\pi} \operatorname{Li}_{2}\left(4 z \sin ^{2} \theta\right) d \theta .
$$

This is an analytic function of $z$. For $|z|<1 / 4$ we may use the original series for $\mathrm{Li}_{2}$ and expand term by term using Wallis' formula to derive

$$
\begin{aligned}
\tau(z) & =\frac{2}{\pi} \sum_{n \geq 1} \frac{(4 z)^{n}}{n^{2}} \int_{0}^{\pi} \sin ^{2 n} \theta d \theta=4{ }_{4} F_{3}\left(\begin{array}{c}
1,1,1, \frac{3}{2} \\
2,2,2
\end{array} \mid 4 z\right) \\
& =4 \operatorname{Li}_{2}\left(\frac{1}{2}-\frac{1}{2} \sqrt{1-4 z}\right)-2 \log \left(\frac{1}{2}+\frac{1}{2} \sqrt{1-4 z}\right)^{2} .
\end{aligned}
$$

The final equality can be obtained in Mathematica and then verified by differentiation. In particular, the final function provides an analytic continuation and so we obtain $\tau(1)=2 \zeta(2)+4 i \mathrm{Cl}_{2}(\pi / 3)$ which yields the assertion.

For (4.9), commencing much as in [16, Theorem 11], we write

$$
\mu_{2}(1+x+y)=\frac{1}{4 \pi^{2}} \int_{-\pi}^{\pi} \int_{-\pi}^{\pi} \operatorname{Re} \log \left(1-2 \sin (\theta) e^{i \omega}\right)^{2} d \omega d \theta .
$$

Let $\alpha=2 \sin \theta$. We consider the inner integral

$$
\rho=\rho(\alpha):=\int_{-\pi}^{\pi}\left(\operatorname{Re} \log \left(1-\alpha e^{i \omega}\right)\right)^{2} d \omega .
$$

For $|\theta| \leq \pi / 6$ we directly apply Parseval's identity to obtain

$$
\rho=\pi \operatorname{Li}_{2}\left(4 \sin ^{2} \theta\right) .
$$


In the remaining case, we write

$$
\begin{aligned}
\rho & =\int_{-\pi}^{\pi}\left\{\log |\alpha|+\operatorname{Re} \log \left(1-\alpha^{-1} e^{i \omega}\right)\right\}^{2} d \omega \\
& =2 \pi \log ^{2}|\alpha|-2 \log |\alpha| \int_{-\pi}^{\pi} \log \left|1-\alpha^{-1} e^{i \omega}\right| d \omega+\pi \operatorname{Li}_{2}\left(\frac{1}{4 \sin ^{2} \theta}\right) \\
& =2 \pi \log ^{2}|2 \sin \theta|+\pi \operatorname{Li}_{2}\left(\frac{1}{4 \sin ^{2} \theta}\right),
\end{aligned}
$$

where we have appealed to Parseval's and Jensen's formulas. Thus,

$$
\mu_{2}(1+x+y)=\frac{1}{\pi} \int_{0}^{\pi / 6} \operatorname{Li}_{2}\left(4 \sin ^{2} \theta\right) d \theta+\frac{1}{\pi} \int_{\pi / 6}^{\pi / 2} \operatorname{Li}_{2}\left(\frac{1}{4 \sin ^{2} \theta}\right) d \theta+\frac{\pi^{2}}{54}
$$

since

$$
\frac{2}{\pi} \int_{\pi / 6}^{\pi / 2} \log ^{2} \alpha d \theta=\mu_{2}\left(1+x+y_{*}\right)=\frac{\pi^{2}}{54}
$$

Now, for $\alpha>1$, the functional equation

$$
\mathrm{Li}_{2}(\alpha)+\mathrm{Li}_{2}(1 / \alpha)+\frac{1}{2} \log ^{2} \alpha=2 \zeta(2)+i \pi \log \alpha
$$

from [18, Equation (6) in Appendix A2.1] gives

$$
\int_{\pi / 6}^{\pi / 2}\left\{\operatorname{Re~Li}_{2}\left(4 \sin ^{2} \theta\right)+\operatorname{Li}_{2}\left(\frac{1}{4 \sin ^{2} \theta}\right)\right\} d \theta=\frac{5}{54} \pi^{3} .
$$

We now combine (4.8), (4.11) and (4.10) to deduce the desired result (4.9).

We are now in a position to prove the desired evaluation of $\mu_{2}(1+x+y)$ as a logsine integral.

Proof of Theorem 4.4. Using Proposition 4.6,

$$
\begin{aligned}
\mu_{2}(1+x+y) & =\frac{\pi^{2}}{36}+\frac{2}{\pi} \int_{0}^{\pi / 6} \operatorname{Li}_{2}\left(4 \sin ^{2} w\right) d w \\
& =\frac{\pi^{2}}{36}+\frac{2}{\pi} \sum_{n \geq 1} \frac{4^{n}}{n^{2}} \int_{0}^{\pi / 6} \sin ^{2 n} w d w \\
& =\frac{\pi^{2}}{36}+\frac{\sqrt{3}}{\pi} \sum_{n \geq 1} 4^{-n}\left(\begin{array}{c}
2 n-1 \\
n-1
\end{array}\right) \sum_{k=n}^{\infty} \frac{1}{(2 k+1)\left(\begin{array}{c}
2 k \\
k
\end{array}\right)}
\end{aligned}
$$

where the last line is a consequence of the formula

$$
\int_{0}^{\pi / 6} \sin ^{2 n} w d w=\frac{\sqrt{3}}{2} 4^{-n}\left(\begin{array}{c}
2 n-1 \\
n-1
\end{array}\right) \sum_{k=n}^{\infty} \frac{1}{(2 k+1)\left(\begin{array}{c}
2 k \\
k
\end{array}\right)}
$$


given in [16]. Hence, on using a beta integral and then exchanging the sum and integral, we obtain

$$
\begin{aligned}
\mu_{2}(1+x+y) & =\frac{\pi^{2}}{36}+\frac{2 \sqrt{3}}{\pi} \sum_{n \geq 1}\left(\begin{array}{c}
2 n-1 \\
n-1
\end{array}\right) \int_{0}^{1 / 2} \frac{t^{n}(1-t)^{n}}{1-t+t^{2}} d t \\
& =\frac{\pi^{2}}{36}+\frac{2 \sqrt{3}}{\pi} \int_{0}^{1 / 2} \sum_{n \geq 1}\left(\begin{array}{c}
2 n-1 \\
n-1
\end{array}\right) \frac{(t(1-t))^{n}}{1-t+t^{2}} d t \\
& =\frac{\pi^{2}}{36}+\frac{\sqrt{3}}{\pi} \int_{0}^{1 / 2} \frac{2 \operatorname{Li}_{2}(t)-\log ^{2}(1-t)}{1-t+t^{2}} d t
\end{aligned}
$$

where the last equality comes from evaluating the power series above.

Further careful integrations by parts let us use [18, Appendix A5.3, (1)] to derive

$$
\begin{gathered}
\pi \mu_{2}(1+x+y)=\frac{67}{324} \pi^{3}+2 \mathrm{Cl}_{2}\left(\frac{\pi}{3}\right) \log 3-8 \operatorname{Im~Li}_{3}(i \sqrt{3}) \\
+4 \operatorname{Im~Li}_{3}\left(\frac{3+i \sqrt{3}}{2}\right) .
\end{gathered}
$$

Next, we note that

$$
\operatorname{Im} \operatorname{Li}_{3}\left(\frac{3+i \sqrt{3}}{2}\right)=\frac{55}{1296} \pi^{3}+\frac{5}{48} \pi \log ^{2} 3+\operatorname{Im~Li}_{3}\left(\frac{3-i \sqrt{3}}{6}\right)
$$

while

$$
\operatorname{Im~Li}_{3}(i \sqrt{3})=\frac{1}{16} \pi^{3}+\frac{1}{16} \pi \log ^{2} 3-\frac{1}{6} \operatorname{Ti}_{3}\left(\frac{1}{\sqrt{3}}\right) .
$$

Above, we have had recourse to various reduction formulae $[6,18]$ for higher Clausen functions to arrive at the final form. Substituting (4.13) and (4.14) in (4.12), we arrive at the asserted result (4.5).

A connection with the log-sine integrals is made by noting that

$$
\begin{aligned}
& \operatorname{Ti}_{3}\left(\frac{1}{\sqrt{3}}\right)=\frac{5}{8} \operatorname{Ls}_{3}\left(\frac{2 \pi}{3}\right)-\frac{1}{2} \operatorname{Ti}_{2}\left(\frac{1}{\sqrt{3}}\right) \log 3-\frac{1}{48} \pi \log ^{2} 3+\frac{2}{27} \pi^{3}, \\
& \operatorname{Ti}_{2}\left(\frac{1}{\sqrt{3}}\right)=\frac{5}{6} \mathrm{Cl}_{2}\left(\frac{\pi}{3}\right)-\frac{\pi}{12} \log 3 .
\end{aligned}
$$

These follow from [18, Equation (44), p. 298 and Equation (18), p. 292] respectively. Applying (4.15) and (4.16) to (4.5) now yields (4.6).

Finally, we observe that it is possible to take the analysis of $\mu_{n}(1+x+y)$ for $n \geq 3$ a fair distance. This will be detailed in the forthcoming paper [3]. 
4.4. Evaluation of $\boldsymbol{\mu}_{\mathbf{2}}(\mathbf{1}+\boldsymbol{x}+\boldsymbol{y}+\boldsymbol{z})$. Paralleling the evaluation of $\mu_{2}(1+x+y)$ in Theorem 4.4, we now give a closed form for $\mu_{2}(1+x+y+z)$ which was obtained in [9] by quite different methods to those of Theorem 4.4.

Theorem 4.7. Define $\lambda_{4}$ as in (1.8). Then

$$
\mu_{2}(1+x+y+z)=\frac{12}{\pi^{2}} \lambda_{4}\left(\frac{1}{2}\right)-\frac{\pi^{2}}{5}
$$

Proof. The formula

$$
\pi^{2} W_{4}^{\prime \prime}(0)=24 \operatorname{Li}_{4}\left(\frac{1}{2}\right)-18 \zeta(4)+21 \zeta(3) \log 2-6 \zeta(2) \log ^{2} 2+\log ^{4} 2
$$

was deduced in [9]. We now observe that

$$
24 \mathrm{Li}_{4}\left(\frac{1}{2}\right)-18 \zeta(4)+21 \zeta(3) \log 2-6 \zeta(2) \log ^{2} 2+\log ^{4} 2=12 \lambda_{4}\left(\frac{1}{2}\right)-\frac{\pi^{4}}{5},
$$

and appeal to Equation (4.1) for $\mu_{2}(1+x+y+z)=W_{4}^{\prime \prime}(0)$.

4.5. A conjecture of Rodriguez Villegas. Finally, we mention two conjectures concerning the Mahler measures $\mu(1+x+y+z+w)$ and $\mu(1+x+y+z+w+v)$, contained in slightly different form in [13]. These correspond to the moment values $W_{5}^{\prime}(0)$ and $W_{6}^{\prime}(0)$.

Recall that $\eta$ is the Dirichlet eta-function given by

$$
\eta(\tau)=\eta(q):=q^{1 / 24} \prod_{n=1}^{\infty}\left(1-q^{n}\right)=q^{1 / 24} \sum_{n=-\infty}^{\infty}(-1)^{n} q^{n(3 n+1) / 2}
$$

where $q=e^{2 \pi i \tau}$. The following two conjectural expressions have been put forth by Rodriguez Villegas:

$$
\mu(1+x+y+z+w) \stackrel{?}{=}\left(\frac{15}{4 \pi^{2}}\right)^{5 / 2} \int_{0}^{\infty}\left\{\eta^{3}\left(e^{-3 t}\right) \eta^{3}\left(e^{-5 t}\right)+\eta^{3}\left(e^{-t}\right) \eta^{3}\left(e^{-15 t}\right)\right\} t^{3} d t
$$

and

$$
\mu(1+x+y+z+w+v) \stackrel{?}{=}\left(\frac{3}{\pi^{2}}\right)^{3} \int_{0}^{\infty} \eta^{2}\left(e^{-t}\right) \eta^{2}\left(e^{-2 t}\right) \eta^{2}\left(e^{-3 t}\right) \eta^{2}\left(e^{-6 t}\right) t^{4} d t .
$$

As discussed in [9], we have confirmed numerically that the evaluation of $\mu(1+x+$ $y+z+w+v)$ in (4.18) holds to 600 places. Likewise, we have confirmed that (4.19) holds to 80 places.

\section{Conclusion}

It is reasonable to ask what other Mahler measures can be placed in log-sine form, and to speculate as to whether the $\eta$ integrals (4.18) and (4.19) can be. 
As described in [17], it is a long-standing question due to Lehmer as to whether $\mu(P)$ can be arbitrarily close to zero for single-variable integer polynomials $P$. For higher Mahler measures, [17, Theorem 7] shows that for $k=1,2, \ldots$ the measure $\mu_{2 k+1}\left(\left(x^{n}-1\right) /(x-1)\right)$ does tend to zero as $n$ goes to infinity.

It was shown in (2.3) that for positive integers $k$,

$$
\pi \mu\left(1+x+y_{1}, 1+x+y_{2}, \ldots, 1+x+y_{k}\right)=\operatorname{Ls}_{k+1}\left(\frac{\pi}{3}\right)-\operatorname{Ls}_{k+1}(\pi) .
$$

This rapidly tends to zero with $k$ since

$$
\left|\operatorname{Ls}_{k+1}\left(\frac{\pi}{3}\right)-\operatorname{Ls}_{k+1}(\pi)\right| \leq \frac{2 \pi}{3} \log ^{k} 2 .
$$

Can one find any natural polynomial sequences so that $\mu\left(P_{n}, Q_{n}\right)$ tends to zero with $n$, and so generalize [17, Theorem 7]?

\section{Acknowledgements}

Thanks are due to Yasuo Ohno and Yoshitaka Sasaki for introducing us to the relevant papers during their recent visit to CARMA. Especial thanks are due to James Wan and David Borwein for many useful discussions. We are also grateful to the reviewer for several helpful suggestions.

\section{References}

[1] H. Akatsuka, 'Zeta Mahler measures', J. Number Theory 129(11) (2009), 2713-2734.

[2] J. M. Borwein, D. H. Bailey and R. Girgensohn, Experimentation in Mathematics: Computational Paths to Discovery, 1st edn (A K Peters, Natick, MA, 2004).

[3] D. Borwein, J. M. Borwein, A. Straub and J. Wan, 'Log-sine evaluations of Mahler measures, II', Integers, in press.

[4] J. M. Borwein, D. M. Bradley, D. J. Broadhurst and P. Lisoněk, 'Special values of multiple polylogarithms', Trans. Amer. Math. Soc. 353(3) (2001), 907-941.

[5] J. M. Borwein, D. J. Broadhurst and J. Kamnitzer, 'Central binomial sums, multiple Clausen values, and zeta values', Experiment. Math. 10(1) (2001), 25-34.

[6] J. M. Borwein, O.-Y. Chan and R. E. Crandall, 'Higher-dimensional box integrals', Experiment. Math. 19(3) (2010), 431-446.

[7] J. M. Borwein and R. E. Crandall, 'Closed forms: what they are and why we care'. Notices Amer. Math. Soc., in press.

[8] J. M. Borwein and A. Straub, 'Special values of generalized log-sine integrals'. Proceedings of ISSAC 2011 (36th International Symposium on Symbolic and Algebraic Computation) San Jose, CA, June 2011 (ACM, New York, 2011), pp 43-50.

[9] J. M. Borwein, A. Straub, J. Wan and W. Zudilin, 'Densities of short uniform random walks (with an appendix by Don Zagier)'. Canadian J. Math., published electronically at http://dx.doi.org/10. 4153/CJM-2011-079-2.

[10] J. M. Borwein, I. J. Zucker and J. Boersma, 'The evaluation of character Euler double sums', Ramanujan J. 15(3) (2008), 377-405.

[11] D. W. Boyd, 'Speculations concerning the range of Mahler's measure', Canad. Math. Bull. 24 (1981), 453-469. 
[12] A. Davydychev and M. Kalmykov, 'New results for the $\varepsilon$-expansion of certain one-, two- and three-loop Feynman diagrams', Nuclear Phys. B 605 (2001), 266-318.

[13] S. Finch, 'Modular forms on $S L_{2}(\mathbb{Z})$ ', Preprint, 2005.

[14] W. Fuchs, 'Two definite integrals (solution to a problem posed by L. Lewin)', Amer. Math. Monthly 68(6) (1961), 580-581.

[15] M. Kalmykov, 'About higher order $\epsilon$-expansion of some massive two- and three-loop masterintegrals', Nuclear Phys. B 718 (2005), 276-292.

[16] N. Kurokawa, M. Lalín and H. Ochiai, 'Higher Mahler measures and zeta functions', Acta Arith. 135(3) (2008), 269-297.

[17] M. Lalín and K. Sinha, 'Higher Mahler measure of cyclotomic polynomials and Lehmer's question', Ramanujan J. 26 (2011), 257-294.

[18] L. Lewin, Polylogarithms and Associated Functions (North Holland, Amsterdam, 1981).

[19] L. Lewin, 'On the evaluation of log-sine integrals', The Mathematical Gazette 42 (1958), 125-128.

[20] F. W. J. Olver, D. W. Lozier, R. F. Boisvert and C. W. Clark, NIST Handbook of Mathematical Functions (Cambridge University Press, Cambridge, 2010).

[21] A. v. d. Poorten, 'A proof that Euler missed... Apéry's proof of the irrationality of $\zeta(3)$ ', Math. Intelligencer 1(4) (1979), 195-203.

[22] Y. Sasaki, 'On multiple higher Mahler measures and multiple $L$ values', Acta Arith. 144(2) (2010), $159-165$.

JONATHAN M. BORWEIN, School of Mathematical and Physical Sciences,

University of Newcastle, Callaghan, NSW 2308, Australia

e-mail: jonathan.borwein@newcastle.edu.au

ARMIN STRAUB, Department of Mathematics, Tulane University, New Orleans, LA 70118, USA

e-mail: astraub@tulane.edu 